\title{
CLINICAL PROFILE OF TOXIC KERATOCONJUNCTIVITIS AFTER OCULAR TRAUMA WITH INSECT
}

\author{
Patel S. ${ }^{1}$, Dhakhwa K. ${ }^{2}$, Rai S.K.C. ${ }^{3}$, Bhattarai B ${ }^{4}$., Pandey A. ${ }^{5}$ Badhu B.P. ${ }^{6}$
}

\begin{abstract}
INTRODUCTION: Toxic keratoconjunctivitis is a frequently encountered problem in the subspecialty of cornea. Toxic keratoconjunctivitis can occur as a complication of exposure to various substances. Toxic keratoconjunctivitis due to exposure to many chemicals and medications have been well described in the literature. The present study was carried out to explore the clinical profile of toxic keratoconjunctivitis caused by insect an unknown cause in a tertiary care center in Lumbini zone of western region of Nepal.
\end{abstract}

METHODS: It was a prospective observational study conducted in cornea clinic of Lumbini Eye Institute, Bhairahawa, Nepal from 1 October 2012 to 30 September 2013. Institutional approval and informed consent was taken. All the patients presented with toxic keratoconjunctivitis caused by insect or unknown causes were included in the study. Patients with other known causes of toxic keratoconjunctivitis like drugs, chemicals were excluded from the study. Detail clinical history, general physical examination and meticulous ophthalmological examination were carried out in all the subjects as per predesigned pro-forma. Patients were followed up after 3 days, 1 week and 2 weeks after initial presentation.

RESULTS: Total 39 patients with toxic keratoconjunctivitis presented in cornea clinic of Lumbini Eye Institute during the study period were enrolled and analyzed. The mean age of the patients was $38.20 \pm 15.44$ year ranging from 16-75 years. Male were affected more than female. In our study 33 (84.62\%) patients were male and only $6(15.38 \%)$ patients were female. Most of patients presented during the month of April and May. Twenty one (53.85\%) of our patients noticed some small insects lodged in the eye. Eighteen (56.15\%) patients had history of exposure of foreign body but could not identify it as insect. Most of patients noticed exposure of insect or an unknown object in Night (36, 92.31\%) and only $3(7.69 \%)$ patients during day time. Mean duration of illness was $2.13 \pm 1.19$ days ranging from 1 to 5 days. Right eye and left eye were involved equally. Complete healing of lesions occurred in all the patients

CONCLUSION: Toxic keratoconjunctivitis caused by insect or unknown foreign body is a frequently encountered problem seen in summer months in a tertiary care center in Lumbini zone of western region of Nepal. With treatment all the patient had good out come in form of complete healing of ocular lesions.

KEY WORDS: Toxic Keratitis, Toxic Kerato-conjunctivitis.

1\&4. Ophthalmologist, Lumbini Eye Institute, Bhairahawa, Nepal

$2 \& 3$. Associate Professor, Department of Ophthalmology, Lumbini Eye Institute, National Academy of Medical Sciences, Kathmandu, Nepal

5. Post Graduate Resident, Department of Ophthalmology, Universal College of Medical Sciences \& Teaching Hospital, Bhairahawa, Nepal

6. Professor, Department of Ophthalmology, B.P.Koirala Institute of Health Sciences, Dharan, Nepal

\author{
For Correspondence \\ Dr. Sushila Patel, M.D., \\ Ophthalmologist, \\ Lumbini Eye Institute, \\ Bhairahawa, Nepal \\ E-mail:drsushilapatel@yahoo.com
}




\section{INTRODUCTION}

Toxic keratoconjunctivitis is a frequently encountered problem in the subspecialty of cornea. ${ }^{1}$ Often in clinical practice, patients present with a red and irritated eye that can not be explained solely on the basis of infection, allergic reaction, dry eye, trauma, or other common causes of external ocular irritation. A toxic substance can be defined as one which by its chemical action causes damage to structure, or a disturbance of function, over and above any therapeutic effect. ${ }^{2}$ Toxic keratoconjunctivitis may occur as a complication of exposure to various substance. Toxic keratoconjunctivitis due to exposure to many chemicals and medications have been well described in the literature. ${ }^{3-6}$ The most common manifestation is a coarse punctuate epithelial keratopathy. Heaped up opaque epithelium, swirl patterns and pseudodendrites may occasionally develop. Toxic ulcerative keratitis is the most serious form of corneal toxicity.

The present study was carried out to study the clinical profile of toxic keratoconjunctivitis caused by insect an unknown cause in a tertiary care center in Lumbini zone of western region of Nepal.

\section{MATERIAL AND METHODS}

It was a prospective observational study conducted in cornea clinic of Lumbini Eye Institute, Bhairahawa, Nepal from 1 October 2012 to 30 September 2013. Institutional approval was taken. All the patients presented with toxic keratoconjunctivits caused by an insect or an unknown cause was included in the study. Patients with other known causes of toxic keratoconjunctivitis like drugs, Chemical, animal venoms were excluded from the study.

After obtaining a written informed consent, detailed clinical history, general physical examination and meticulous ophthalmological examination was carried out in all the patients as per predesigned pro-forma. History regarding onset of various symptoms like pain, redness, photophobia, watering, discharge and diminution of vision was taken. Visual acuity of both the eyes was measured with Snellen chart. Detailed corneal examination was done. The corneal epithelial defect and superficial puntate keratitis was seen after staining with fluorescein using the graduated slit beam of a Haag-Streit 900 slit lamp. All the patients were treated with fortified cefazolin 5\%, preservative free lubricants, antibiotic ointments and low dose steroids like fluorometholone. Patients with large epithelial defects were treated by applying contact bandage lens. Patients were followed up after 3 days, 1 week and after 2 weeks from date of presentation.
Clinical response had been evaluated in the form of visual acuity, healing of corneal defect and complications if any at each follow up visits. Treatment outcome had been evaluated in form of signs of healing, final visual acuity achieved, surgical intervention required or not and complication if any. The collected data were entered into Microsoft Excel Spreadsheet and analyzed using SPSS ver 11.5 (PC) / EpiInfo (CDC, Atlanta, GA, USA). Means and standard deviation of age of the patients and duration of illness was calculated.

\section{RESULTS}

Total 39 patients with toxic keratoconjuctivitis presented in cornea clinic of Lumbini eye Institute during the study period were enrolled and analyzed. The mean age of the patients was $38.20 \pm 15.44$ years ranging from $16-75$ years. There were patients from all age group. (Table 1)

Table 1: Age distribution of patients

\begin{tabular}{|c|c|}
\hline Age Group & Numbers (\%) \\
\hline $16-25$ & $11(28.20 \%)$ \\
\hline $26-35$ & $9(23.08 \%)$ \\
\hline $36-45$ & $8(20.51 \%)$ \\
\hline $46-55$ & $7(17.95 \%)$ \\
\hline $56-65$ & $1(1.67 \%)$ \\
\hline $66-75$ & $3(7.69 \%)$ \\
\hline
\end{tabular}

Male were affected more than female. In our study 33 $(84.62 \%)$ patients were male and only $6(15.38 \%)$ patients were female. Most of our patients were from surrounding districts of Nepal and neighboring Indian state of Uttar Pradesh. Twenty three (58.97\%) patients were from Nepal and $16(41.03 \%)$ patients were from Indian state of Uttar Pradesh. Majority of the patients were from Rupendehi $(19,48.71 \%)$ and Maharajgunj $(13,33.33 \%)$ district. Rests were from Nawalparasi (3, 7.69\%), Kapilvastu (1, 2.56\%), Kushinagar (1, 2.56\%), Gorakhpur (1, 2.56\%) and Balrampur (1, 2.56\%). Majority of the patients were from rural areas. Twenty five $(64.10 \%)$ were from rural areas and $14(35.9 \%)$ were from urban areas. There were $16(41.02 \%)$ agricultural workers, 6 (15.38\%) students, 6 (15.38\%) servicemen, 5 (12.82\%) housewives, 5 (12.82\%) shopkeepers and 1 (2.56\%) carpenter. Most of patients presented during the month of April and May. There were $25(64.1 \%)$ patients presented in April, 8 (20.5\%) in May, $2(5.12 \%)$ in June and 1 (2.56\%) each in September, November, December and March. Most of patients noticed exposure of insect or an unknown object in Night $(36,92.31 \%)$ and only $3(7.69 \%)$ patients during day time. Twenty one $(53.85 \%)$ of our patients notice some small insects lodged in the eye. Eighteen $(56.15 \%)$ patient had history of exposure of foreign body but could not identify it as insect. 
Mean duration of illness was $2.13 \pm 1.19$ days ranging from 1 to 5 days. Right eye and left eye were involved equally. Right eye was involved in $20(51.29 \%)$ patients and left eye was involved in $19(48.71 \%)$ patients. There was no difference in the two eyes in terms of laterality. Conjunctival reaction, chemosis, lid or periorbital swelling, punctate epithelial erosion was seen in mildest form. (Figure 1) Which healed within one week of presentation without any scar. (Figure 2) Diffuse punctate epitheliopathy was observed in severe cases (Figure 3).

\section{Figure 1: Mild form of toxic reaction}

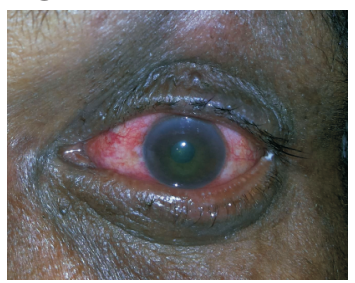

Figure 2: Improvement after 7 days of therapy

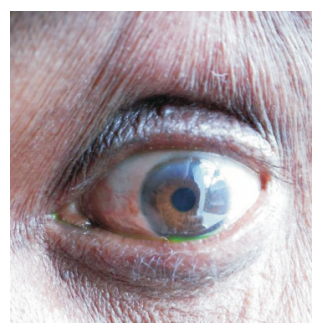

Figure 3: Severe form of toxic reaction

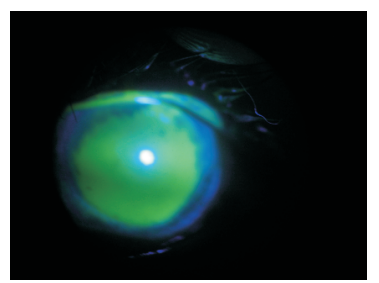

At the time of presentation visual acuity was taken with the help of Snellen chart and repeated during each visit. Vision in the involved eye varied from 6/6 to CF. Most of the patients presented with low vision which improved during subsequent follow up. (Table 2)

\section{Table 2: Vision of involved eye}

\begin{tabular}{|c|c|c|c|c|}
\hline Vision of involved eye & First visit & $\mathbf{3}^{\text {rd }}$ day & 1 Week & 2 Week \\
\hline $6 / 6-6 / 12$ & $3(7.69 \%)$ & $14(35.89 \%)$ & $28(71.79 \%)$ & $31(79.49 \%)$ \\
\hline $6 / 18-6 / 36$ & $15(38.46 \%)$ & $14(35.89 \%)$ & $8(20.51 \%)$ & $1(2.56 \%)$ \\
\hline $6 / 60-1 / 60$ & $18(46.15 \%)$ & $11(28.20 \%)$ & $3(7.69 \%)$ & $2(5.12 \%)$ \\
\hline CF & $3(7.69 \%)$ & 0 & 0 & 0 \\
\hline
\end{tabular}

Five patients who had marked improvement in $2^{\text {nd }}$ follow up did not come in $3^{\text {rd }}$ follow up. Contact bandage lens was applied in three cases due to large epithelial defect. Two patients in whom vision could not improve to $6 / 60$ were found to have brown cataract.

\section{DISCUSSION}

Classification systems for corneal toxicity are by disease, route of exposure and time course, or by agent ${ }^{7,8}$ The cornea may be involved alone or with the conjunctiva. Corneal toxicity is caused by chemical trauma, for which diagnosis is seldom a problem. The clinical signs of toxic keratoconjunctivits due to drugs are usually nonspecific and identical to those resulting from other causes of corneal epithelial disease such as punctate keratopathy, coarse focal keratopathy, pseudodendrites, filamentary keratopathy, and persistent epithelial defect. More specific signs occur with the preservative phenylmercuric nitrate toxicity, ${ }^{7}$ which may produce band keratopathy. Cumulative drug deposition is because of systemic medications (amiodarone, indomethacin, chloroquine) causing deposits within the corneal epithelium ${ }^{7}$ that are usually of little functional significance. The topical anaesthetics and the more toxic antivirals (idoxuridine and trifluorothymidine) are among the more commonly abused drops. ${ }^{9}$ Toxic keratopathy may result from abuse of topically administered anesthetics even at a very low concentration. ${ }^{10}$ Benzalkonium chloride (BAC), a cationic detergent, is the most commonly used preservative in topical ophthalmic preparations. It is a positively charged surface active alkylamine biocide, which interacts with high affinity with membrane proteins such as guanine nucleotide triphosphate binding proteins ( $\mathrm{G}$ proteins), affecting signal transduction in a variety of cell types and processes..$^{11}$ Toxic keratoconjunctivitis due to insect injuries are rare. Bee and Wasp stings to the cornea and/ or conjunctiva are well documented. ${ }^{12}$ The medically important groups of Hymenoptera are the Apoidea (bees), Vespoidea (wasps, hornets, and yellow jackets), and Formicidae (ants). These insects deliver their venom by stinging their victims. Acutely, conjunctival hyperemia and chemosis usually occurs, sometimes associated with severe pain, corneal oedema with subsequent decrease in vision. The variability of response depends in the quantity of the venom injected. Diagnosis of bee and wasp stings stem from a history of potential contact matched with onset of appropriate clinical signs. In our study most of patient had history of exposure to small insect in the eye, but they were unable to identify it. Most of the injuries occurred in night and after exposure they were unable to open the eye due to foreign body sensation.

Corneal epithelial disruption and stromal ulceration without a history of contact lens wear, trauma, surgery, predisposing keratopathy or immunodeficiency warrants thorough investigation of patient social habits, including substance abuse. ${ }^{13}$ 


\section{CONCLUSION}

Toxic keratoconjunctivitis caused by insect or unknown foreign body is a frequently encountered problem seen in summer months in a tertiary care center in Lumbini zone of western region of Nepal. Most of the patients in our study had exposure with insect in night. With treatment all the patient had good out come in form of complete healing of ocular lesions. Further studies are required to identify species of insects responsible for this condition. It will further help in the development appropriate strategyfor it's prevention.

\section{REFERENCES}

1. Albert DM, Jakobiec FA, et al. Principles and Practice of Ophthalmology. 2nd ed. Vol. 1-6. Philadelphia : W.B. Saunders, 2000: p. 793.

2. Wilson FM. Adverse external ocular effects of topical ophthalmic medications. Surv Ophthalmol 1979;24:68.

3. Gasset AR. Benzalkonium chloride toxicity to the human cornea. Am JOphthalmol 1977;84:169-71.

4. Gasset AR, Ishii Y, Kaufman HE, et al. Cytotoxicity of ophthalmic preservatives. Am JOphthalmol 1974;78:98-105.

5. Means TL, Holley GP, Mehta KR, et al. Corneal edema from an intraocular irrigating solution containing benzalkonium chloride. J Toxicol Cut Ocul Toxicol 1994; 13:67-81.
6. Edelhauser HF, Van Horn DL, Schultz RO, et al. Comparative toxicity of intraocular irrigating solutions on the corneal endothelium. Am JOphthalmol 1976;81:473-81.

7. Grant WM. Toxicology of the Eye. Charles C Thomas: Springfield, IL, 1986.

8. Duke-Elder S, McFaul PA. Injuries (Part 2). Non-Mechanical Injuries, Vol XIV, System of Ophthalmology. Henry Kimpton: London, 1972.

9. Rocha G, Brunette I, Francois MLe. Severe toxic keratopathy secondary to topical anaesthetic abuse. Can J Ophthalmol 1995; 30: 198-202.

10. Chen HT, Chen KH, Hsu WM. Cornea. Toxic keratopathy associated with abuse of low-dose anesthetic: a case report. 2004 Jul;23(5):527-9.

11. Patarca R, Fletcher MA. Effects of benzalkonium salts on eukaryotic and microbial G-protein-mediated processes and surface membranes. Crit Rev Oncog 1995; 6:327-56.

12. Wiwanitkit V. Corneal wasp sting. Cutan Ocul Toxicol. 2012 Sep;31(3):262.

13. Pilon AF, Scheiffle J. Ulcerative keratitis associated with crack-cocaine abuse. Cont Lens Anterior Eye. 2006 Dec;29(5):263-7. 